UR-1576

Jul 1999

rev. Oct 1999

\title{
On Neutrino Masses and Mixings from Extra Dimensions
}

\author{
Ashok Das and Otto C. W. Kong * \\ Department of Physics and Astronomy, \\ University of Rochester, Rochester NY 14627-0171
}

\begin{abstract}
In the framework of a Kaluza-Klein type theory with the Standard Model fields localized on a 4-dimensional section while gravity propagates in a full $4+\delta$-dimensional space-time, we examine a mechanism of naturally small neutrino mass generation through couplings of the Standard Model singlet fermion(s) living also in the full space-time. A numerical study is carried out on the charged current universality constraint from the ratio of pion decay partial widths. The bounds obtained on the fundamental mass scale could be stringent.
\end{abstract}

Typeset using REVTEX

*Present address: Insitute of Physics, Academia Sinica, Taipei, Taiwan 11529. E-mail: kongcw@phys.sinica.edu.tw . 


\section{INTRODUCTION}

Interests in Kaluza-Klein (KK) type theories with extra space-time dimensions has recently been revived with an odd twist — the usual Standard Model (SM) fields with its chiral fermionic content are assumed to be localized on a 4-dimensional section. With only gravity propagating in the full $4+\delta$-dimensional space-time, the extra dimensions could be "large", with a fundamental mass scale $M_{*}$ as low as a TeV [1,2]. The scenario seems to promise rich phenomenological features, which has been the subject of many recent studies [3]. However, present collider bounds on $M_{*}$ are typically in the TeV range, while it has been shown that astrophysical and cosmological processes provide much stronger bounds [2.4], rendering collider phenomenology uninteresting. For $\delta=2$, the best bound is about $150 \mathrm{TeV}$; while it is about $30 \mathrm{TeV}$ for $\delta=3$. As collider experiments are unlikely to yield information on the feasibility of the specific KK scenario, it would be interesting to have other probes into the extra dimensions. Here in this letter, we illustrate how neutrino physics may just provide us with that.

The problem of neutrino mass generation under such a scenario was discussed in Refs. [5.66]. The relatively small $M_{*}$ value invalidates the popular seesaw mechanism and its various variations. However, as the so-called right-handed neutrino is a SM singlet fermion, it may very well live in the full $4+\delta$ dimensions along with gravity. Naturally small Yukawa couplings to the SM neutrinos can then result from a volume factor. The suppression, given by $M_{*} / M_{P l}$ is roughly in the right range to account for neutrino oscillations [7]. In this letter, we present a careful study of the neutrino mass generation as well as stringent bounds on $M_{*}$ obtained from charged current universality.

\section{A SINGLET FERMION IN 5 DIMENSIONS}

We consider here, for illustrative purposes only, the simple case of one SM singlet fermion $\Psi$ in a 5 -dimensional theory, the latter with co-ordinates $\left(x^{\mu}, y\right)$, with $\mu=0, \cdots, 3$ and the $y$ 
direction compactified on a circle of circumference $2 \pi R$ by making the periodic identification $y \sim y+2 \pi R$. Conventional SM fields are restricted to live on the 4-dimensional section at $y=0$. We adopt the representation where the 5 -D gamma matrices are given by

$$
\Gamma^{\mu}=\left(\begin{array}{cc}
0 & \sigma^{\mu} \\
\bar{\sigma}^{\mu} & 0
\end{array}\right), \quad \Gamma^{4}=\left(\begin{array}{cc}
i & 0 \\
0 & -i
\end{array}\right) .
$$

This matches with the 4 -D chiral representation in Ref. [8] for $\Gamma^{\mu}=\gamma^{\mu}$ and $\Gamma^{4}=i \gamma^{5}$. The fermion $\Psi$ is a 4 -spinor which decomposes as

$$
\Psi=\left(\begin{array}{c}
i \psi_{L} \\
\psi_{R}
\end{array}\right)
$$

where each component has a 4-D chirality marked by its eigenvalue under $\gamma^{5}$; and a phase $i$ is introduced for later convenience. When compactifying the fifth dimension, the Fourier expansion

$$
\Psi(x, y)=\sum_{n=-\infty}^{\infty} \frac{1}{\sqrt{2 \pi R}} \Psi_{n}(x) e^{i n y / R}
$$

applies componentwise, with the resultant 2-spinors $\psi_{L n}$ and $\psi_{R n}$ liable to be interpreted as KK towers of independent 4-D Weyl spinors. From the $y$-component of the 5-D free action $\bar{\Psi} i \Gamma^{\alpha} \partial_{\alpha} \Psi$, we have

$$
\begin{aligned}
S_{\Psi} & =-\int d^{4} x d y \bar{\Psi} \gamma^{5} \partial_{y} \Psi \\
& =\int d^{4} x \frac{n}{R}\left(\psi_{L n}^{\dagger} \psi_{R n}+\psi_{R n}^{\dagger} \psi_{L n}\right)
\end{aligned}
$$

Next, we consider admissible interactions of the components of $\Psi$ with the SM fields living at $y=0$. Recall that the SM leptonic doublet $L$ is a Weyl (2-)spinor, conventionally taken to be left-handed. Here we keep careful track of the spinor structures by sticking to 2-spinor notations and paying attention to the difference between $\psi$ and $\psi^{\dagger}$. We have

$$
S^{\mathrm{int}}=\int d^{4} x\left(\frac{\lambda_{\nu}}{\sqrt{M_{*}}} \psi_{R}^{\dagger}(x, y=0) \phi^{*}(x) L(x)+\frac{\lambda_{\nu}^{\prime}}{\sqrt{M_{*}}} \psi_{L}^{c \dagger}(x, y=0) \phi^{*}(x) L(x)+\text { h.c. }\right) \text {, }
$$

where the factor $\sqrt{M_{*}}$ is introduced to make the Yukawa couplings $\lambda$ and $\lambda^{\prime}$ pure numbers; $\phi$ denotes the Higgs scalar doublet; and the superscript $c$ indicates a 2-spinor obtained from 
the charge conjugation of the original 2-spinor. In particular, $\psi_{L}^{c}=i \sigma^{2} \psi_{L}^{*}$, and transforms as a right-handed 2-spinor. Note that we violate the conventional notation here [9].

With electroweak symmetry breaking, the above interaction generates 4-D quadratic fermionic terms of the same form

$$
m \psi_{R n}^{\dagger} \nu_{L}+m^{\prime} \psi_{L n}^{c \dagger} \nu_{L}
$$

for each $n$. From Eqs.(4) and (6), we obtain a Majorana mass matrix for the effective 4-D neutral fermions (neutrinos) of the form

$$
\mathcal{L}_{\text {mass }}=\frac{1}{2} N^{c} \mathcal{M} N^{T}
$$

given by

$$
\mathcal{M}=\left(\begin{array}{cccccccc}
0 & m & m^{\prime} & m & m^{\prime} & m & m^{\prime} & \ldots \\
m & 0 & 0 & 0 & 0 & 0 & 0 & \cdots \\
m^{\prime} & 0 & 0 & 0 & 0 & 0 & 0 & \cdots \\
m & 0 & 0 & 0 & 1 / R & 0 & 0 & \cdots \\
m^{\prime} & 0 & 0 & 1 / R & 0 & 0 & 0 & \cdots \\
m & 0 & 0 & 0 & 0 & 0 & 2 / R & \cdots \\
m^{\prime} & 0 & 0 & 0 & 0 & 2 / R & 0 & \cdots \\
\vdots & \vdots & \vdots & \vdots & \vdots & \vdots & \vdots & \ddots
\end{array}\right)
$$

with

$$
\begin{gathered}
N=\left(\nu_{L}, \psi_{R 0}^{c}, \psi_{L 0}, \psi_{R 1}^{c}, \psi_{L 1}, \psi_{R 2}^{c}, \psi_{L 2}, \cdots\right) \\
N^{c}=\left(\nu_{L}^{c \dagger}, \psi_{R 0}^{\dagger}, \psi_{L 0}^{c \dagger}, \psi_{R 1}^{\dagger}, \psi_{L 1}^{c \dagger}, \psi_{R 2}^{\dagger}, \psi_{L 2}^{c \dagger}, \cdots\right) .
\end{gathered}
$$

Notice that we have used general relations of the form

$$
\psi_{1}^{c \dagger} \psi_{2}^{c}=\psi_{2}^{\dagger} \psi_{1}
$$

The part of the above mass matrix involving the $n$-th KK states has a universal structure which allows us to introduce the following compressed notation, 


$$
\mathcal{M}=\left(\begin{array}{ccccc}
0 & m & m^{\prime} & m & m^{\prime} \\
m & 0 & 0 & 0 & 0 \\
m^{\prime} & 0 & 0 & 0 & 0 \\
m & 0 & 0 & 0 & n / R \\
m^{\prime} & 0 & 0 & n / R & 0
\end{array}\right) .
$$

Here, it is to be understood that the columns and rows with the $n / R$ represents a repeated structure for each $n$; the latter is always restricted to be non-zero, but have both signs. The notation can be used for the more general cases with more dimensions, to be discussed below.

Assuming a hierarchy $m, m^{\prime} \ll 1 / R$, it is straight forward to work out through a block perturbative analysis the "seesaw" contributions of the heavy $n(\neq 0)$ states to the first three (light) states of $\mathcal{M}$. We have, from the compressed notation of $\mathcal{M}$ above, the simple result

$$
-\left(\begin{array}{ccc}
\frac{2 m m^{\prime}}{n / R} & 0 & 0 \\
0 & 0 & 0 \\
0 & 0 & 0
\end{array}\right) .
$$

It is easy to see that for the full $\mathcal{M}$ matrix, as explicitly given by Eq.(8), contributions from each $n$ simply adds up to give the only non-zero entry as $\sum_{n} \frac{2 m m^{\prime}}{n / R}$. It indicates that only the (Majorana) mass of $\nu_{L}$, the SM neutrino, receives perturbative contributions, which is what one should expect from the mixing terms. The perturbative effect from each $n$ is small and the perturbative assumption seems not to be upset, yet the total sum goes as $\sum_{n} 1 / n$ which diverges naively as $\ln n$, suggesting that the neutrino mass becomes infinite! Actually, however, as the Fourier expansion in Eq.(3) contains both positive as well as negative $n$ modes, the $\sum_{n} \frac{2 m^{\prime}}{n / R}$ is always zero (There is no $n=0$ term in the sum). As we will show explicitly below, in a somewhat more complicated scenario, a simple perturbational analysis gives a first order correction to the light mass value $m$ of order $\sum_{n} \frac{m^{2} R^{2}}{n^{2}}$, the same form as the case with a pure Dirac mass matrix discussed in Ref. [5]. The latter corresponds to setting $m^{\prime}=0$. It is important to note here that with both $m$ and $m^{\prime}$ non-zero, the neutrino 
mass matrix cannot be consistently written in the Dirac form. The sum to infinity of $\frac{1}{n^{2}}$ is still divergent for $\delta \leq 2$, but will be well-behaved when properly truncated.

Note that when the compactification is performed on a $Z_{2}$ orbifold, about half of the KK modes are projected out. The surviving $\psi_{R n}$ modes are to be taken as the linear combinations of the positive and negative $n$ modes for each $n$ which are even under a $y$-reflection; while the surviving $\psi_{\text {Ln }}$ modes are the corresponding linear combinations which are odd under the $y$-reflection. In particular, the $\psi_{L_{0}}$ would be projected out and hence decoupled in $\mathcal{M}$. The surviving $\psi_{L n}$ modes actually have zero amplitude at the SM world $(y=0)$ and $m^{\prime}$ has to be set to zero.

The admission of both $m$ and $m^{\prime}$ [both terms in Eq.(5)] is interesting when more than one family of SM neutrinos are considered. The two 2-spinors in $\Psi$ can then give mass to two neutrinos with mixing. Let us now consider such masses and mixings for $\nu_{e}$ and $\nu_{\mu}$. Eq.(10) is modified as $\mathcal{M}$ is extended to include a second $\nu_{L}$. It then takes the block form

$$
\mathcal{M}=\left(\begin{array}{ccc}
0 & \Omega_{o}^{T} & \Omega_{o}^{T} \\
\Omega_{o} & 0 & 0 \\
\Omega_{o} & 0 & M_{n}
\end{array}\right),
$$

where $\Omega_{o}$ is a general $2 \times 2$ matrix representing mass terms of the form given in Eq.(6), but now with two $\nu_{L}$ 's, and $M_{n}=\left(\begin{array}{cc}0 & n / R \\ n / R & 0\end{array}\right)$. Skipping the algebraic details, we simply note that a unitary transformation can bring $\mathcal{M}$ to the form

$$
\mathcal{M}=\left(\begin{array}{cc}
\left(\begin{array}{cc}
m_{1} & 0 \\
0 & m_{2}
\end{array}\right) & \Omega^{T} \\
0 & \left(\begin{array}{cc}
-m_{1} & 0 \\
0 & -m_{2}
\end{array}\right) \\
-\Omega & -\Omega^{T} \\
\Omega & \left(\begin{array}{cc}
n / R & 0 \\
0 & -n / R
\end{array}\right)
\end{array}\right),
$$

where $\Omega$ may be taken as a general $2 \times 2$ matrix, and $m_{1}$ and $m_{2}$ are the eigenvalues of $\Omega_{o}$ taken as a Dirac mass matrix. Our compressed notation involving the $n \neq 0 \mathrm{KK}$ modes is still 
effective. Note that each pair of the light states having equal and opposite mass eigenvalues can be considered as a Dirac state consisting of a $\nu_{L}$ state and a linear combination of the zero modes from $\Psi$; each of the pair of (Majorana) states is then an equal admixture of the latter.

Entries in $\Omega$ in the above form of $\mathcal{M}$ are of the same order as $m_{1}$ and $m_{2}$ or that of the entries in $\Omega_{o}$, hence $\ll 1 / R$. The diagonal entries of $\mathcal{M}$ are, therefore, the approximate mass eigenvalues. We can perform a direct perturbative analysis to find out the first order corrections to the light masses, as well as the mixings between the $n \neq 0 \mathrm{KK}$ modes and the light states, hereafter denoted by $\left|\nu_{i}^{ \pm}\right\rangle$(for diagonal mass entry $\pm m_{i}, i=1$ or 2 ). Let $U$ denote the unitary transformation that diagonalizes $\mathcal{M}$ in Eq.(13). Perturbation analysis gives, in our compressed notation,

$$
\begin{aligned}
U_{+i, \pm n} & =\frac{-\omega_{ \pm i}}{ \pm n / R-m_{i}} \\
U_{-i, \pm n} & =\frac{\omega_{ \pm i}}{ \pm n / R+m_{i}}
\end{aligned}
$$

where we have used the convenient notations of the original states labeled by the indexsequence $\{+1,+2,-1,-2,+n,-n\}$ (the first four states are not to be confused with the KK states with $n= \pm 1$ or 2 ) and $\Omega=\left(\begin{array}{cc}\omega_{+1} & \omega_{+2} \\ \omega_{-1} & \omega_{-2}\end{array}\right)$. Perturbations to masses of the four light states are given by

$$
\begin{aligned}
\Delta m_{ \pm i} & =\sum_{n} \frac{-\omega_{+i}^{2}}{n / R \mp m_{i}}+\sum_{n} \frac{-\omega_{-i}^{2}}{-n / R \mp m_{i}} \\
& \simeq \mp m_{i} R^{2}\left(\omega_{-i}^{2}+\omega_{+i}^{2}\right) \sum_{n} \frac{1}{n^{2}} .
\end{aligned}
$$

\section{GENERALIZATION TO HIGHER DIMENSIONS}

The case with one "large" extra dimension is known to be unrealistic [1, 2]. For $\Psi$ living in $4+\delta$ dimensions with $\delta=2$ or 3 , the gamma matrices are $8 \times 8$. There is a Weyl 4 -spinor for $\delta=2$, while the smaller spinor representation, for $\delta=3$ or 4 , is an 8-spinor. Nevertheless, 
complications of the sort do not change the general pattern. The higher dimensional spinor $\Psi$ can always be decomposed into KK towers of 4-D 2-spinors with Dirac mass couplings as in the 5-D case above. Assuming the size of the extra dimensions are all the same, the major difference is that the $\mathrm{KK}$ towers now form a $\delta$-dimensional integral lattice, and we get four 2-spinors for $\delta=3$ or 4 and eight 2-spinors for $\delta=5$ or 6 . Hence for $\delta \geq 3$, one $\Psi$ singlet can already give masses and mixings to all three SM neutrinos. For the purpose of the analysis in this letter, we will stick to the two 2-spinor formulation discussed above. Generalization to the other cases is straight forward though a bit tedious.

With $\delta$ extra dimensions, $n$ in our compressed notation should be generalized to mean the magnitude of a vector within the $\delta$-dimensional integral lattice,

$$
n=\sqrt{n_{1}^{2}+\ldots \ldots+n_{\delta}^{2}}
$$

there would be a KK mode, with the corresponding column and row entries to $\mathcal{M}$, for each of such vectors, and $\sum_{n}$ would be a summation over all of them. A $\sum_{n} \frac{1}{n^{2}}$ is then always divergent. However, if the KK tower is truncated at an ultraviolet cutoff $\sim M_{*}$, a sum of the form $\sum_{n} \frac{m^{2} R^{2}}{n^{2}}$, when approximated by an integral over the $n$-lattice, goes like

$$
\frac{S_{\delta}}{\delta-2} \frac{\lambda_{\nu}^{2} v^{2}}{2 M_{*}^{2}}=\frac{2 \pi^{\delta / 2}}{\Gamma(\delta / 2)} \frac{1}{(\delta-2)} \frac{\lambda_{\nu}^{2} v^{2}}{2 M_{*}^{2}},
$$

where $S_{\delta}$ is the result of a $\delta$-dimensional angular integration and $v=246 \mathrm{GeV}$. The result is always $\ll 1$. Recall that

$$
m=\frac{\lambda_{\nu} v}{\sqrt{2}} \frac{M_{*}}{M_{P l}},
$$

and

$$
R^{\delta} \simeq M_{P l}^{2} / M_{*}^{\delta+2}
$$

Hence, adopting the truncation procedure, the validity of the perturbative results above is well justified.

We want to have a word of caution here about the KK tower truncation. The general theory of "large" extra dimensions presumes a fundamental scale $M_{*}$ and hence an ultraviolet 
cut-off, which has been generally taken by previous authors as implying a truncation of the KK tower of states. However, in the situation discussed here, where the higher dimensional $\Psi$ has mass mixing with the 4-D fields (neutrinos), it is not a priori clear whether the truncation should rather not be applied to the physical states. The truncation of the KK tower in $\Psi$, and hence the mass matrix $\mathcal{M}$ before diagonalization, is however crucial for the above perturbation results to make sense. Without the truncation, the mass eigenvalue perturbations have $\sum_{n} 1 / n^{2}$ divergences. In fact, the infinite number of $n$ states would impose an infinite normalization factor on the matrix elements $U_{ \pm i, \pm n}$ which is neglected in Eq.(14). The normalizations then make each non-zero elements in $U$ infinitesimally smaller. The physical consequence of the latter scenario is stunning. Any SM field localized in 4-D having mass mixing with higher dimensional fields will be a linear combination of an infinite number of mass eigenstates most of them lying beyond the cut-off scale and truncated from the physical spectrum; hence, any SM coupling to the field has effectively vanishing couplings to full physical spectrum of states involving the field. In particular, applying to the neutrino mixing scenario, leptonic $W$-boson decays would be vanishing as effective total amplitude into a physical lepton together with any physical "neutrino" within the threshold is infinitesimally small.

\section{CONSTRAINT FROM VIOLATION OF CHARGED CURRENT UNIVERSALITY}

With the word of caution above, we go on in this section to discuss constraint from the violation of charged current universality as a result of the neutrino masses and mixings, taking the usual assumption of KK tower truncation. We will concentrate on charged pion decay through $\Gamma(\pi \rightarrow e \nu) / \Gamma(\pi \rightarrow \mu \nu)$. The analysis involved in the case is relatively straight forward while the process is likely to provide the most stringent constraint due to the good experimental results and that the small $\pi$ mass makes the quantity extremely sensitive to heavy neutrino states involved. Ref. [10] has presented such a discussion, which we, however, 
do not consider to be quite complete as the effect of the heavier, KK tower states were not incorporated. Though we stick here to the more general formulation with both types of terms in Eq.(5) admitted, our result holds even when only $\psi_{R}$ coupling is allowed, which is the case discussed in Ref. [10] following Ref. [5]. For more background information and related analysis of charged current universality, readers are referred to Ref. [11].

As discussed above, each SM neutrino $\nu_{L}$ is now given by a linear combination of various mass eigenstates; it is predominately an equal mixture of a Dirac pair of mass about $\pm m_{i}$, with small admixture from the heavier physical states of masses about $\pm n / R$. We have, for charged lepton $\ell_{i}$,

$$
\begin{aligned}
\Gamma\left(\pi \rightarrow \ell_{i} \nu\right) \propto & \frac{1}{2}\left[\left(1-\sum_{n}\left|U_{+i, n}\right|^{2}-\sum_{n}\left|U_{+i,-n}\right|^{2}\right) P_{0}^{\pi \ell_{i}}+\sum_{n}\left(\left|U_{+i,+n}\right|^{2}+\sum_{n}\left|U_{-i, n}\right|^{2}\right) P_{n}^{\pi \ell_{i}}\right. \\
& \left.+\left(1-\sum_{n}\left|U_{-i, n}\right|^{2}-\sum_{n}\left|U_{-i, n}\right|^{2}\right) P_{0}^{\pi \ell_{i}}+\sum_{n}\left(\left|U_{-i, n}\right|^{2}+\sum_{n}\left|U_{-i,-n}\right|^{2}\right) P_{n}^{\pi \ell_{i}}\right],
\end{aligned}
$$

where

$$
P_{n}^{\pi \ell_{i}}=\theta\left(m_{\pi}-m_{\ell_{i}}-m_{\nu_{n}}\right)\left[\frac{m_{\ell_{i}}^{2}}{m_{\pi}^{2}}+\frac{m_{\nu_{n}}^{2}}{m_{\pi}^{2}}-\left(\frac{m_{\ell_{i}}^{2}}{m_{\pi}^{2}}-\frac{m_{\nu_{n}}^{2}}{m_{\pi}^{2}}\right)^{2}\right] \lambda^{1 / 2}\left(1, \frac{m_{\ell_{i}}^{2}}{m_{\pi}^{2}}, \frac{m_{\nu_{n}}^{2}}{m_{\pi}^{2}}\right),
$$

is the matrix element-phase space function, with $\lambda(a, b, c)=(a-b-c)^{2}-4 b c$, and $m_{\nu_{n}}^{2} \simeq$ $n^{2} / R^{2}$ being the square of the physical neutrino masses; $P_{0}^{\pi \ell_{i}}$ is a bit of abuse of notation as the physical mass $\pm m_{i}$ is safely approximated as zero. We have shown in the above formula only the dependence on the partial width of the masses and mixings. Should all the physical neutrino masses be negligible, there would, of course, be no change in the decay width. The important point to note here is that the KK tower contributes to arbitrarily heavy neutrino states up to the ultraviolet cutoff beyond the $\pi$-decay threshold. We rewrite the expression in the form

$$
\Gamma\left(\pi \rightarrow \ell_{i} \nu\right) \propto P_{0}^{\pi \ell_{i}}\left(1-\sum_{n}^{\left(\pi-\ell_{i}\right)^{\prime}} \frac{\left(\omega_{+i}^{2}+\omega_{-i}^{2}\right) R^{2}}{n^{2}}+\sum_{n}^{\pi-\ell_{i}} \frac{\left(\omega_{+i}^{2}+\omega_{-i}^{2}\right) R^{2}}{n^{2}} \mathcal{P}_{n}^{\pi \ell_{i}}\right)
$$

where we have substituted Eq.(14) with $m_{i}$ neglected, and introduced

$$
\mathcal{P}_{n}^{\pi \ell_{i}}=\frac{P_{n}^{\pi \ell_{i}}-P_{0}^{\pi \ell_{i}}}{P_{0}^{\pi \ell_{i}}}
$$


whereas the summation $\sum_{n}^{\pi-\ell_{i}}$ indicates a sum up to the $\pi$-decay threshold, i.e. $|n| \leq R\left(m_{\pi}-\right.$ $m_{\ell_{i}}$ ) and the summation $\sum_{n}^{\left(\pi-\ell_{i}\right)^{\prime}}$ goes from there to the ultraviolet cutoff $|n| \leq R M_{*}$. We further simplify the expression to

$$
\Gamma\left(\pi \rightarrow \ell_{i} \nu\right) \propto P_{0}^{\pi \ell_{i}}\left[1+\left(\lambda_{+i}^{2}+\lambda_{-i}^{2}\right) \frac{R^{2} v^{2} M_{*}^{2}}{2 M_{P l}^{2}}\left(\sum_{n}^{\pi-\ell_{i}} \frac{1}{n^{2}} \mathcal{P}_{n}^{\pi \ell_{i}}-\sum_{n}^{\left(\pi-\ell_{i}\right)^{\prime}} \frac{1}{n^{2}}\right)\right]
$$

where we introduced, in accordance with Eq.(16),

$$
\omega_{ \pm i}=\frac{\lambda_{ \pm i} v}{\sqrt{2}} \frac{M_{*}}{M_{P l}} .
$$

Here, $\lambda_{ \pm i}$ 's can be considered as Yukawa couplings in some chosen basis.

In the above expressions, we have implicitly assumed the charged leptons $\ell_{i}$ 's are physical states. Actually, there should be a leptonic-CKM matrix involved as well. However, to the extent that we have no information about the original Yukawa couplings ( $\lambda$ 's) as given in Eqs.(5) and (16), we can do no better than stating that Eq.(22) applies to $e$ and $\mu$ each with $\left(\lambda_{+i}^{2}+\lambda_{-i}^{2}\right)$ replaced by a corresponding quantity dependent on the original Yukawa couplings. Hence, we write

$$
\frac{\Gamma(\pi \rightarrow e \nu)}{\Gamma(\pi \rightarrow \mu \nu)}=R_{\pi \mu}^{\pi e} \frac{\left[1+Y_{e} \frac{R^{2} v^{2} M_{*}^{2}}{2 M_{P l}^{2}}\left(\sum_{n}^{\pi-e} \frac{1}{n^{2}} \mathcal{P}_{n}^{\pi e}-\sum_{n}^{(\pi-e)^{\prime}} \frac{1}{n^{2}}\right)\right]}{\left[1+Y_{\mu} \frac{R^{2} v^{2} M_{*}^{2}}{2 M_{P l}^{2}}\left(\sum_{n}^{\pi-\mu} \frac{1}{n^{2}} \mathcal{P}_{n}^{\pi \mu}-\sum_{n}^{(\pi-\mu)^{\prime}} \frac{1}{n^{2}}\right)\right]},
$$

where $Y_{e}$ and $Y_{\mu}$, of the same order as the $\lambda^{2}$ 's, parametrize our ignorance about the Yukawa couplings as pointed out above, and

$$
R_{\pi \mu}^{\pi e}=1.233 \times 10^{-4}
$$

is the SM result. The experimental number is $(1.230 \pm 0.004) \times 10^{-4}$ at one $\sigma$. With only $\psi_{R}$ allowed to coupled, an extra $\psi_{R}$ has to be used to replace $\psi_{L}^{c}$, but the formula here for $\frac{\Gamma(\pi \rightarrow e \nu)}{\Gamma(\pi \rightarrow \mu \nu)}$ applies unchanged.

To check the explicit modification of the $\pi$-decay ratio, and hence the corresponding constraint on the "large" extra dimension scenario, we evaluate the relevant terms in the above equation, approximating the summation by an integration. Some numerical results 
are listed in Table 1. Reading from the table, we can draw lower bounds on $M_{*}$ using the experimental result, for various number of extra dimensions. The kind of bounds obtained gives only an order of magnitude. The first reason for this is our ignorance in $Y_{e}$ and $Y_{\mu}$, which potentially can be improved by other complimentary analysis such as fitting neutrino oscillation data. A deeper problem comes from our ignorance of the ultraviolet regime. We have used a simple cutoff at $M_{*}$ to truncate the KK tower. In the numerical calculation, we have implemented Eq.(17) with an equality, which in term can be considered as our definition of $M_{*}$ itself. However, the numerical result is not too sensitive to the explicit cutoff value used; the $\sum_{n}^{\pi-\ell}$ terms are not dependent on it while the $\sum_{n}^{(\pi-\ell)^{\prime}}$ terms are dominated by small n. For example, we have checked explicitly that changing the cutoff by an order does not change our numbers in any substantial way.

As shown in Table 1, the $\sum_{n}^{\pi-\mu} \frac{1}{n^{2}} \mathcal{P}_{n}^{\pi \mu}$ term is the smallest one in Eq.(24), never playing a significant role. This is mainly a result of the very small threshold, and the generic small value of $\mathcal{P}_{n}^{\pi \mu}$. On the contrary, $\mathcal{P}_{n}^{\pi e}$ can reach very large value for $n / R$ (neutrino mass) in the $\mathrm{MeV}$ region. There is a strong suppression from the $\left|U_{ \pm i, \pm n}\right|^{2}$ prefactors $[c f . \mathrm{Eq}(18)]$, but also a huge number of relevant states contributing. For $\delta=2$, the $\sum_{n}^{\pi-e} \frac{1}{n^{2}} \mathcal{P}_{n}^{\pi e}$ term dominates and gives large modifications to $\Gamma(\pi \rightarrow e \nu)$. In that case, the numerical value of the term scales as $M_{*}^{-\delta}$, to a very good approximation. From the table, we can see that this implies a lower bound of roughly $M_{*}<1600 \mathrm{TeV}$, assuming $Y_{e}$ to be order one. For $\delta>2$, however, $1 / R$ increases pretty fast which results in a strong suppression in the term. The two $\sum_{n}^{(\pi-\ell)^{\prime}}$ terms go as $M_{*}^{-2}$ for all $\delta>2$ and become dominating. The two terms tend to cancel one another, with final result dependent on $Y_{e}-Y_{\mu}$. Taking the latter to be order one, with $Y_{\mu}>Y_{e}$, gives roughly $M_{*}<20 \mathrm{TeV}$ with a very weak dependence on $\delta$. In fact,

$$
\frac{R^{2} v^{2} M_{*}^{2}}{2 M_{P l}^{2}} \sum_{n}^{(\pi-\ell)^{\prime}} \frac{1}{n^{2}} \simeq \frac{\pi^{\delta / 2}}{\Gamma(\delta / 2)} \frac{1}{(\delta-2)} \frac{v^{2}}{M_{*}^{2}},
$$

when the lower limits of summation are neglected, which serves as a good approximation for all the $\delta \leq 3$ cases as suggested by the apparent identical results in the $\sum_{n}^{(\pi--)^{\prime}}$ and $\sum_{n}^{(\pi-\mu)^{\prime}}$ ( $c f$. third and last columns of Table I). Hence we refrain from presenting explicit number 
for $\delta>4$.

A word of caution is in order for the interpretation of the results. While taking the $\lambda$-type couplings to be not substantially smaller than unity is a common strategy among most previous authors on the subject, a suppression in their values would weaken the constraints obtained very effectively. As stated above, the constraints go as the squares of the $\lambda$ 's. Hence, suppressed $\lambda$ values would easily render the constraints obtained much less interesting. From the theoretical point of view, assuming order one couplings avoids the need of an extra mechanism to produce the otherwise suppressed values. Hence, the strategy is commonly adopted. Phenomenologically speaking, however, one would have to check if there are numbers that can fit in with all the experimental data, through a comprehensive analysis of all aspects of neutrino physics from a specific model. That is a task beyond the present work.

\section{CONCLUDING REMARKS}

Neutrino physics is rather subtle and unconventional in a theory with extra dimensions not seen by the other SM fields. While singlet fermion(s) living in at least some of the extra dimension(s) and coupling to the SM neutrinos seems to provide a mechanism to naturally explain the small neutrino masses, a comprehensive analysis of all aspects of neutrino phenomenology still needs to be performed. We have discussed here only some of the issues involved and presented numerical results from the pion decay constraints. Assuming order one Yukawa couplings, our results give a $1600 \mathrm{TeV}$ bound on $M_{*}$ for two extra dimensions, substantially stronger than any other known constraints. The high $M_{*}$ value also implies larger neutrino mass for the lightest states, threatening fittings with neutrino oscillations

[7]. For more than two extra dimensions, the bound stays around $20 \mathrm{TeV}$. It should be emphasized again that the above strong numerical bounds are based on order one Yukawa couplings, which is pushing the limit of validity of the perturbational analysis used. More realistic couplings, say 0.3 , actually give a bound no stronger than the existing bounds which 
can be found in Ref. [4.

The bounds obtained here do not necessarily rule out smaller $M_{*}$, however, if an alternate neutrino mass generation mechanism is assumed. Results would also be modified if the singlet fermion(s) sees some, instead of all, of the extra dimensions that gravity sees. In any case, we expect various charged current universality constraints to be very important, compared with other more direct collider bounds. Neutrino physics may provide the best probe into the extra dimensions, if they exist.

We would like to thank G. Shiu and G.-H. Wu for discussions. This work was supported in part by the U.S. Department of Energy, under grant DE-FG02-91ER40685 


\section{REFERENCES}

[1] N. Arkani-Hamed, S. Dimopoulos and G. Dvali, Phys. Lett. B429 (1998) 263; I. Antoniadis, N. Arkani-Hamed, S. Dimopoulos and G. Dvali, Phys. Lett. B436 (1998) 257.

[2] N. Arkani-Hamed, S. Dimopoulos and G. Dvali, Phys. Rev. D59 (1999) 086004.

[3] There is a long list of papers on the subject, T. Han, J. Lykken, and R.J. Zhang, Phys. Rev. D59 (1999) 105006; and G.F. Giudice, R. Rattazzi, and J.D. Wells, Nucl. Phys. B544 (1999) 3; for instance, give discussions of the formulations and Feynman rules, and some basic results. Since we consider the type of analysis of limited interest here, we will refrai from listing further references.

[4] S. Nussinov and R. Shrock, Phys. Rev. D59 (1999) 105002; S. Cullen and M. Perelstein, SLAC-PUB-8084, hep-ph/9903422; L.J. Hall and D. Smith, LBNL-43091, hepph/9904267; V. Barger, T. Han, C. Kao, and R.J. Zhang, MADPH-99-1118, hepph/9905474.

[5] N. Arkani-Hamed, S. Dimopoulos, G. Dvali, and J. March-Russell, SLAC-PUB-8014, hep-ph/9811448.

[6] K.R. Dienes, E. Dudas and T. Gherghetta, CERN-TH-98-370, hep-ph/9811428.

[7] G. Dvali and A.Yu. Smirnov, NYU-TH-99-3-03, hep-ph/9904211.

[8] M.E. Peskin and D.V. Schroeder, An Introduction to Quantum Field theory, Addison Wesley, 1995.

[9] P. Ramond, Field Theory — A Modern Primer, Benjamin/Cummings, 1981.

[10] A.E. Faraggi and M. Pospelov, TPI-MINN-99/4, hep-ph/9901299, to be published in Phys. Lett. B.

[11] O.L.G. Peres, V. Pleitez, and R. Zukanovich Funchal, Phys. Rev. D 50 (1994) 513; M.M. Guzzo, O.L.G. Peres, V. Pleitez, and R. Zukanovich Funchal, Phys. Rev. D 53 (1996) 
2851; A. Bottino, N. Fornengo, C.W. Kim, and G. Mignola, Phys. Rev. D 53 (1996) 6361; M. Bisset, O.C.W. Kong, C. Macesanu, and L.H. Orr, UR-1524, hep-ph/9811498.

\section{Table Caption:-}

Table I: Numerical Results on terms contributing to deviation of $\frac{\Gamma(\pi \rightarrow e \nu)}{\Gamma(\pi \rightarrow \mu \nu)}$ from the Standard Model value, as given by Eq.(24).

Table I :-

\begin{tabular}{|c|c|c|c|c|c|}
\hline \multicolumn{2}{|c|}{$\delta M_{*}(\mathrm{TeV})$} & $\frac{R^{2} v^{2} M_{*}^{2}}{2 M_{P I}^{2}} \sum_{n}^{\pi-e} \frac{1}{n^{2}} \mathcal{P}_{n}^{\pi e}$ & $\frac{R^{2} v^{2} M_{*}^{2}}{2 M_{P}^{2}} \sum_{n}^{(\pi-)^{\gamma}} \frac{1}{n^{2}}$ & $\frac{R^{2} v^{2} M_{*}^{2}}{2 M_{P}^{2}} \sum_{n}^{\pi-\mu} \frac{1}{n^{2}} \mathcal{P}_{n}^{\pi \mu}$ & $\frac{R^{2} v^{2} M_{*}^{2}}{2 M_{P}^{2}} \sum_{n}^{(\pi-\mu)^{\top}} \frac{1}{n^{2}}$ \\
\hline \multirow[t]{3}{*}{2} & 1 & $7.52 \pi \times 10^{2}$ & $5.37 \pi \times 10^{-1}$ & $-6.74 \pi \times 10^{-3}$ & $6.23 \pi \times 10^{-1}$ \\
\hline & 100 & $7.52 \pi \times 10^{-2}$ & $8.16 \pi \times 10^{-5}$ & $-8.47 \pi \times 10^{-7}$ & $9.02 \pi \times 10^{-5}$ \\
\hline & 1600 & $2.94 \pi \times 10^{-4}$ & $3.84 \pi \times 10^{-7}$ & $-3.31 \pi \times 10^{-9}$ & $4.18 \pi \times 10^{-7}$ \\
\hline \multirow[t]{3}{*}{3} & 1 & $9.60 \pi \times 10^{-2}$ & $1.21 \pi \times 10^{-1}$ & $-4.79 \pi \times 10^{-7}$ & $1.21 \pi \times 10^{-1}$ \\
\hline & 10 & $9.60 \pi \times 10^{-5}$ & $1.21 \pi \times 10^{-3}$ & $-4.79 \pi \times 10^{-10}$ & $1.21 \pi \times 10^{-3}$ \\
\hline & 20 & $1.20 \pi \times 10^{-5}$ & $3.03 \pi \times 10^{-4}$ & $-5.99 \pi \times 10^{-11}$ & $3.03 \pi \times 10^{-4}$ \\
\hline \multirow[t]{3}{*}{4} & 1 & $3.66 \pi^{2} \times 10^{-6}$ & $3.03 \pi^{2} \times 10^{-2}$ & $-6.99 \pi^{2} \times 10^{-12}$ & $3.03 \pi^{2} \times 10^{-2}$ \\
\hline & 10 & $3.66 \pi^{2} \times 10^{-10}$ & $3.03 \pi^{2} \times 10^{-4}$ & $-6.99 \pi^{2} \times 10^{-16}$ & $3.03 \pi^{2} \times 10^{-4}$ \\
\hline & 20 & $2.29 \pi^{2} \times 10^{-11}$ & $7.56 \pi^{2} \times 10^{-5}$ & $-4.37 \pi^{2} \times 10^{-17}$ & $7.56 \pi^{2} \times 10^{-5}$ \\
\hline
\end{tabular}

